\title{
PREVALENCIA DE AGENTES TRANSMISIBLES POR TRANSFUSION Y FACTORES ASOCIADOS EN UN BANCO DE SANGRE DE CORDOBA- COLOMBIA 2014-2016
}

\section{PREVALENCE OF TRANSMISSIBLE AGENTS BY TRANSFUSION AND ASSOCIATED FACTORS IN A BLOOD BANK FROM CÓRDOBA-COLOMBIA 2014-2016}

\author{
Leyand Ruiz Mendoza ${ }^{1}$, Rossana Villegas Gracia ${ }^{2}$, Jaiberth Cardona Arias ${ }^{3}$
}

\begin{abstract}
${ }^{1}$ Bacteriologa y Laboratorista Clínica, Magister en Salud Pública. Bacterióloga en Banco de Sangre de Córdoba LTDA, Calle 22 No. 6-1, Montería, Córdoba, Colombia, e-mail: leyand16@gmail.com, (DiDhttps://orcid.org/0000-0001-9667-7064; ${ }^{2}$ Bacterióloga, Magister en Microbiología y Bioanálisis énfasis hematología, Docente de planta. Universidad de Córdoba, Carrera 11A No.62B-72, Montería-Córdoba, Colombia, e-mail: rvillegasgracia@correo.unicordoba.edu.co, (iDhttps://orcid.org/00000001-5475-7365; ${ }^{3}$ Microbiólogo y Bioanalista, Magíster Epidemiología, Magíster Economía aplicada, Estudiante Doctorado en Salud Pública, Docente asociado. Universidad de Antioquia. Investigador Faculta de Medicina. Universidad Cooperativa de Colombia, Calle 70 No. 52 - 51, Bloque 5, oficina 103, Medellín, Colombia, e-mail: jaiberthcardona@gmail.com, (iDhttps:// orcid.org/0000-0002-7101-929X
\end{abstract}

Rev. U.D.C.A Act. \& Div. Cient. 21(2):Julio-Diciembre 2018 https://doi.org/10.31910/rudca.v21.n2.2018.969

Artículo de acceso abierto publicado por Revista U.D.C.A Actualidad \& Divulgación Científica bajo una licencia Creative Commons CC BY-NC 4.0

\section{RESUMEN}

La terapia transfusional es una importante alternativa terapéutica, pero el riesgo de transmitir infecciones por esta vía constituye un problema clínico y de salud pública, con magnitud y factores asociados heterogéneos, según la población de referencia de cada banco de sangre. El objetivo del estudio fue determinar la prevalencia de agentes transmisibles por transfusión y factores asociados en un banco de sangre de Córdoba-Colombia 2014-2016. Se realizó un estudio de prevalencia en la totalidad de donantes del banco de sangre, se estimó la prevalencia global de reactividad y específica por agente. Los factores asociados, se estudiaron con chi cuadrado, razones de prevalencia y razones de odds, ajustados a un modelo de regresión logística multivariante. Se incluyeron 39.825 donantes. La prevalencia global de positividad fue 1,4\%. La prevalencia para Treponema pallidum fue $0,43 \%$; Tripanosoma cruzi, 0,39\%; virus de la hepatitis B core, $0,32 \%$ y Antígeno de Superficie de la Hepatitis B, 0,05\%; Virus de la Inmunodeficiencia Humana, 0,14\%; Virus Linfotrópico de Células T Humana, 0,08\%; Virus de la Hepatitis C, 0,04\% y Plasmodium spp., 0\%. En la prevalencia global, las infecciones por Treponema pallidum, positividad para el anticuerpo contra el antígeno central del virus de hepatitis B y Virus Linfotrópico de Células T Humana, fueron estadísticamente mayor en mujeres, personas de mayor edad, residentes de otros departamentos diferente de Córdoba, amas de casa, pensionados, agricultores y ganaderos, administradores y otras profesiones, militares y trabajadores de la salud. La prevalencia de infecciones fue baja; sin embargo, el hallazgo de subgrupos con prevalencias estadísticamente significativa permite orientar nuevas investigaciones e intervenciones de vigilancia epidemiológica.

Palabras clave: bancos de sangre, transfusión sanguínea, infecciones, prevalencia (Thesaurus: DeCS Descriptores en Ciencias de la salud).

\section{ABSTRACT}

Transfusion therapy is an important therapeutic alternative; the risk of transmitting infections in this way constitutes a clinical and public health problem, with magnitude and associated heterogeneous factors according to the reference population of each blood bank. The objective of the study was to determine the prevalence of transmissible agents by transfusion and associated factors in a blood bank of Córdoba-Colombia 2014-2016. A prevalence study was carried out in all blood bank donors, the global prevalence of reactivity was estimated and the agent was specified, the associ- 
ated factors were studied with chi-square, prevalence ratios and odds ratios adjusted to a regression model. multivariate logistics. 39,825 donors were included. The overall prevalence of positivity was $1.4 \%$. The prevalence for Treponema pallidum was $0.43 \%$, Trypanosoma cruzi $0.39 \%$, hepatitis B virus core $0.32 \%$ and Hepatitis B Surface Antigen 0.05\%, Human Immunodeficiency Virus $0.14 \%$, Human T-cell Lymphotropic Virus $0.08 \%$, Hepatitis C Virus $0.04 \%$ and Plasmodium spp. $0 \%$.In the overall prevalence, $T$. pallidum infections, positivity for the antibody against the central antigen of the hepatitis virus B and Lymphotropic Human T Cell Virus were statistically higher in women, older people, residents of other departments different from Córdoba, housewives, pensioners, farmers and ranchers, administrators and other professions, military and health workers. The prevalence of infections was low, however, the finding of subgroups with statistically significant prevalences allows to guide new research and epidemiological surveillance interventions.

Keywords: blood bank, blood transfusion, infections, prevalence.

\section{INTRODUCCIÓN}

La terapia transfusional es considerada una importante alternativa terapéutica en caso de accidentes, traumatismos, complicaciones en embarazos, pacientes con enfermedades onco-hematológicas, entre otras situaciones, que generan pérdida excesiva de la sangre. A pesar de los múltiples avances en medicina transfusional, no se dispone de una fuente diferente a la humana para obtener hemocomponentes que suplan las necesidades de los pacientes, garantizando una alta seguridad (Organización Mundial de la Salud, 2010).

En este orden de ideas, las Infecciones Transmisibles por Transfusión (ITT) constituyen una complicación de gran importancia en relación con la morbilidad y la mortalidad de los receptores de sangre y un problema de salud pública, por la transmisión potencial de agentes virales, bacterianos y parasitarios (Giraldo-Valencia et al. 2015). La transfusión de una unidad de sangre contaminada con diferentes agentes infecciosos, como el virus de la inmunodeficiencia humana $(\mathrm{VIH})$, el virus Linfotrópico de células T(HTLV), Virus de las hepatitis B (VHB) y C (VHC), Treponema pallidum, Plasmodium o Trypanosoma cruzi, deriva en diversos problemas para la salud del receptor, al tiempo que se relaciona con un elevado riesgo de transmisión de estos microorganismos a la población general (OMS, 2005).

Pese a la implementación de diferentes normativas colombianas, como la resolución 0901 de 1996, que establece la tamización obligatoria de cinco marcadores infecciosos (Ministerio de Salud Pública República de Colombia, 1996) y la resolución 0437 del 2014, que adiciona la detección de
HTLV I/II y anticuerpos contra el core del virus de la hepatitis B (Ministerio de Salud y Protección Social República Colombia, 2014), existen cuatro posibles razones que aumentan la probabilidad de transmitir estas infecciones en el banco de sangre: i) el período de ventana inmunológica, en el cual, las pruebas serológicas son negativas en un donante infectado; ii) los donantes portadores crónicos y asintomáticos con pruebas serológicas negativas; iii) la presencia de cepas atípicas o mutantes y iv) los errores técnicos en el laboratorio (Blejer et al. 2002).

Dada la importancia de estas infecciones, se han llevado a cabo diversas investigaciones en los bancos de sangre, con el objetivo de determinar su prevalencia entre los donantes. Entre estas, se destaca un estudio realizado en África central, en el 2015, en el cual, se encontró que las prevalencias fueron de $7,83 \%$, para $\mathrm{VIH} ; 10,01 \%$, para $\mathrm{VHB} ; 3,71 \%$, para $\mathrm{VHC}$ y $21,51 \%$, para T. pallidum. La seroprevalencia fue mayor en donaciones por reposición (Xie et al. 2015).

En Venezuela, en el 2007, se reportaron prevalencias para Anticuerpos contra el virus de la Hepatitis B (Anti-HBc), de 11,52\%; Antígeno de Superficie de la Hepatitis B(HBsAg), de 2,52\%; VHC, de 0,56\%; T. cruzi, 0,28\%; T. pallidum, 8,71\% y de 0\%, para VIH y Plasmodium spp. (Suárez et al. 2007). En Perú, para el 2014, la prevalencia global fue de $2,4 \%$, siendo el agente infeccioso más prevalente el VHB, con un $1,44 \%$, seguido de T. pallidum, con el 0,72\% (Concepción Zabaleta et al. 2014).

En Medellín, Colombia, en el 2012, se determinó una prevalencia global de agentes infecciosos de 3,3\%, en donantes de sangre, siendo más frecuente T. pallidum, con un $0,6 \%$, seguido de VHB, con un $0,1 \%$ y 0\%, para VHC, VIH y Chagas, con diferencias estadísticas en la prevalencia de VHB y $T$. pallidum, según sexo y tipo de donante (Patiño Bedoya et al. 2012). Otro estudio en esta misma ciudad, en 2015, reportó asociación estadística de la infección global y específica por T. pallidum con el sexo, el grupo etario, el tipo de donante y la ocupación (Giraldo Valencia et al. 2015), mientras que una investigación en Bogotá presentó una seroprevalencia de Chagas del 0,27\% y entre los positivos, el $48,30 \%$, registró coinfección con Anti-HBc y el 40,38\%, con sífilis (Cruz \& Moreno, 2016).

En adición a los estudios de prevalencia, la Red Nacional de Bancos de Sangre anualmente expone la frecuencia de los agentes infecciosos, en el nivel nacional y departamental (Instituto Nacional de Salud, 2017). Para el 2016, en Colombia, la reactividad a partir de sangre total fue de $0,21 \%, 0,12 \%$, 1,32\%, 0,37\%, 0,27\%, 1,30\%, 0,25\%, para VIH, HBsAg, Anti$\mathrm{HBc}$, VHC, Anti-T. cruzi, T. pallidum, HTLV I/II, respectivamente. A pesar que estas prevalencias dan una pauta sobre las unidades que se están descartando o difiriendo en los 
bancos, no permite tomar decisiones claras con relación al comportamiento epidemiológico, ya que los análisis por subgrupos se limitan a variables, como la edad y el tipo de donante, sin dar cuenta de otras variables relevantes, como el sexo, la ocupación, la frecuencia de donación, entre otras.

Los antecedentes expuestos revelan la diversidad en la magnitud del problema y en los factores asociados con la distribución de agentes infecciosos, tanto en términos de su prevalencia global como la prevalencia específica, según el sexo, el grupo etario, el grupo sanguíneo, el lugar de residencia, la ocupación y el tipo de donación. Conocer la prevalencia de marcadores infecciosos en la población donante por subgrupos permitirá crear estrategias que busquen la fidelización de donantes de bajo riesgo y la educación en los grupos de mayor riesgo, para con ello, disminuir la serorreactividad y disminuir la probabilidad de transmisión de agentes infecciosos. El objetivo de esta investigación fue determinar la prevalencia de agentes transmisibles por transfusión y factores asociados en un banco de sangre en Córdoba-Colombia, durante el 2014 y 2016.

\section{MATERIALES Y MÉTODOS}

Tipo de estudio: Descriptivo transversal, con la totalidad de donantes registrados en un banco de sangre de Montería, Córdoba, durante el 2014 y 2016. En total, se incluyeron 39.825 donantes, en quienes se tomaron, como criterios de inclusión, los requisitos para ser donantes de sangre, según la guía para la selección de donantes de sangre en Colombia, del Instituto Nacional de Salud (2013). Los criterios de exclusión fueron: información incompleta en los resultados de la tamización o en la confirmación diagnóstica y datos incoherentes, como el registro de edad menor de 18 años.

En la recolección de la información, se utilizó, como fuente secundaria, la base de datos proveniente del software e-delphyn $\AA$ del banco de sangre, particularmente, las variables sexo, edad, grupo sanguíneo, lugar de residencia, ocupación (de acuerdo con la Organización Internacional del Trabajo OIT, 1958) y tipo de donante.

La tamización de los marcadores de infección, se realizó por tecnologías distintas; cabe resaltar que, a pesar del cambio del método, la pertinencia de la estimación de la frecuencia de reactividad, sin discriminar por tipo de técnica diagnóstica, se debe al hecho que la sensibilidad de los diferentes métodos fue mayor al 99\%, por lo que no se presentan diferencias estadísticas en las cifras reportadas en los resultados, situación similar a la reportada en las pruebas confirmatorias, que presentan sensibilidad del $100 \%$, con excepción de HTLV, que es del 97,1\% (Tabla 1).
Para la descripción de las características de la población, se calcularon frecuencias absolutas y relativas y, para la edad, medidas de resumen. Se realizó la estimación de frecuencia de reactividad para cada uno de los marcadores de infección con la prueba tamiz, para evidenciar la magnitud de las pérdidas de unidades de sangre en el banco y con la prueba confirmatoria, para evidenciar la "verdadera" ocurrencia de las infecciones en la población de estudio, así como la prevalencia global de infección, todas con su intervalo de confianza del 95\%. La comparación de la prevalencia de cada marcador y de la prevalencia global de infección con las variables cualitativas nominales, como el sexo, la hemoclasificación, el lugar de residencia, la ocupación y el tipo de donación, se realizó con la prueba chi cuadrado de Person; la comparación con el grupo etario, se hizo con la prueba chi cuadrado de tendencia lineal, para variables ordinales. En adición a lo anterior, se calculó la prevalencia específica de infección, según las variables descritas previamente y se estimó la fuerza de asociación con razones de prevalencia y sus intervalos de confianza del 95\%. Vale precisar que la razón de prevalencia es la medida de asociación epidemiológica adecuada para este tipo de estudios, cuando se desea comparar prevalencias específicas e identificar los grupos con una mayor magnitud del evento; en este estudio, se estimó tomando, como grupo de referencia, a aquel que registró la menor prevalencia de infección o el primer grupo, en el caso de variables politómicas y ordinales.

Para determinar si las asociaciones bivariadas eran reales o generadas por un efecto confusor, se hicieron modelos de regresión logística binaria, en los cuales, se introdujeron todas las variables independientes, con valores $p$, menores a 0,25 en el análisis bivariado (criterio de Hosmer y Lemeshow). Estas, solo se aplicaron a la infección global, sífilis, core del VHB, HTLV I/II, ya que fueron las que presentaron un número de infectados suficiente para este tipo de análisis. Los análisis estadísticos, se realizaron en SPSS versión 21.1 y Epidat 3.1; se consideraron significativos valores inferiores a 0,05 .

Consideraciones éticas: El estudio, se considera una investigación sin riesgo, según la resolución colombiana 8430 de 1993 del Ministerio de Salud. Además, para el uso de la información secundaria, se tuvo presente las consideraciones de la resolución 1995. Finalmente, cada donante autorizó el uso de la información, con fines investigativos.

\section{RESULTADOS Y DISCUSIÓN}

La edad media de la población de estudio fue $35,1 \pm 10,5$; el 50\% de los donantes tenía 34 años o menos, el 50\% de los valores centrales de la edad osciló entre 26 y 42 años y el rango entre 18 y 68. De los 39.825 donantes, la mayoría eran hombres (88,7\%), residentes en Montería $(68,9 \%)$, de 
Tabla 1 . Sensibilidad y especificidad de las pruebas de tamización y confirmatorias.

\begin{tabular}{|l|c|c|}
\hline Pruebas de tamización & Especificidad \% & Sensibilidad \% \\
\hline BioelisaHBsAg 3.0 & 99,5 & 100 \\
\hline Bioelisa Anti-HBC & 99,2 & 100 \\
\hline Bioelisa HCV 4.0 & 99,6 & 100 \\
\hline Bioelisa HIV 1+2 4.0 & 99,7 & 100 \\
\hline BioelisaSyphilis 3.0 & 99,8 & 99,4 \\
\hline Bioelisa Chagas & 99,5 & 100 \\
\hline Bioelisa HTLV 1+2 5.0 & 99,8 & 100 \\
\hline ADVIA Centaur HBsAg II & 99,5 & 100 \\
\hline HBc Total (HBcT) ADVIA Centaur & 99,7 & 100 \\
\hline HCV ADVIA Centaur (aHCV) & 99,9 & 100 \\
\hline HIV Ag/Ab Combo (CHIV) & 99,7 & 100 \\
\hline ADVIA Centaur SYPH (Syphilis SYPH) & 99,5 & 99,6 \\
\hline ARCHITEC HBsAgQualitative II & 99,5 & 100 \\
\hline ARCHITEC Anti-HBc II & $\geq 99,5$ & 100 \\
\hline ARCHITEC Anti-HCV & 99,6 & 99,1 \\
\hline ARCHITEC HIV Ag/Ab Combo & $\geq 99,5$ & 100 \\
\hline ARCHITEC Syphilis TP & $\geq 99$ & 100 \\
\hline ARCHITEC Chagas & $\geq 99,5$ & $\geq 99,0$ \\
\hline ARCHITEC HTLV I/II & $\geq 99,5$ & 100 \\
\hline Pruebas confirmatorias & \multicolumn{2}{|c|}{} \\
\hline MurexHBsAg Versión 3 & $>99,5$ & 100,0 \\
\hline LIAISON XL Anti-HBc & 99,9 & 100,0 \\
\hline HCV BLOT 3.0 Western blot assay & 99,5 & 100,0 \\
\hline HIV BLOT 2.2 Western blot assay & 97,1 & 100,0 \\
\hline Syphilis RPR Test & 92,5 & 100,0 \\
\hline Chagas LineBlotlgG & \multicolumn{2}{|c|}{} \\
\hline HTLV BLOT 2.4 Western blot assay & 97,1 \\
\hline
\end{tabular}

Tabla 2. Prevalencia de marcadores de infección en la población de estudio.

\begin{tabular}{|l|c|c|c|c|}
\hline \multirow{2}{*}{ Marcador } & \multicolumn{2}{|c|}{ Prueba de tamización } & \multicolumn{2}{c|}{ Prueba confirmatoria } \\
\cline { 2 - 5 } & Reactividad \% (n) & IC 95\% & Prevalencia (n) & IC 95\% \\
\hline VIH & $0,32(127)$ & $0,26-0,38$ & $0,14(54)$ & $0,10-1,17$ \\
\hline VHB- HbsAg & $0,14(56)$ & $0,10-0,18$ & $0,05(20)$ & $0,03-0,07$ \\
\hline VHB (Anti-HBc) & $2,62(1044)$ & $2,46-2,78$ & $0,32(127)$ & $0,26-0,38$ \\
\hline VHC (anti-VHC) & $0,33(132)$ & $0,27-0,39$ & $0,04(18)$ & $0,02-0,07$ \\
\hline HTLV I/II & $0,54(216)$ & $0,47-0,62$ & $0,08(31)$ & $0,05-0,11$ \\
\hline Trypanosoma cruzi & $0,43(170)$ & $0,36-0,49$ & $0,39(154)$ & $0,32-0,45$ \\
\hline Treponema pallidum & $2,24(894)$ & $2,10-2,39$ & $0,43(170)$ & $0,36-0,49$ \\
\hline Global & $6,25(2488)$ & $6,00-6,49$ & $1,4(574)$ & $1,32-1,56$ \\
\hline
\end{tabular}


ocupación operarios $(43,9 \%)$. Con relación a la hemoclasificación, el grupo más frecuente fue el $\mathrm{O}$ positivo $(61,2 \%)$; el $95 \%$ de los donantes fueron voluntarios y el $5 \%$ de reposición. Con respecto al tipo de donación, en datos establecidos por la Organización Panamericana de la Salud (OPS) con relación al porcentaje de colecta de sangre de donaciones voluntarias en el 2015, Colombia fue el tercer país con mayor porcentaje, con un 91,14\% (Organización Panamericana de la Salud, 2017). El incremento de la donación voluntaria en el país, se puede deber a la ardua labor de educación y campañas encaminadas a generar conciencia y favorecer la captación de donantes voluntarios, además de la sensibilización de la comunidad sobre mantenimiento de estilos de vida saludables y disminución de factores de riesgo. Estos resultados concuerdan con los datos del INS, que registran donaciones voluntarias para el 2016, de un 91,2\% (Instituto Nacional de Salud, 2017).

Con relación al sexo, los resultados de este trabajo, difieren considerablemente con un estudio realizado en Medellín, Colombia, en el que la frecuencia de donación en los hombres fue de 51,5\% (Giraldo Valencia et al. 2015). En India, la proporción de varones donantes fue de $89,96 \%$ y la de mujeres, de 10,04\%. Estos resultados, se pueden deber al temor de algunas mujeres de experimentar posibles reacciones vasovagales (mareos), problemas en el acceso venoso y diferimientos por baja hemoglobina, debido a las pérdidas fisiológicas de sangre, que ocurren en el ciclo menstrual (Mandal \& Mondal, 2016).

El grupo de edad más frecuente fue el comprendido entre 21 y 30 años, lo que se puede relacionar con el hecho que en este se llevan a cabo las compañas extramurales en universidades y en empresas, lo que concuerda con lo reportado por el INS, en el 2016, indicando que, en el departamento de Córdoba, la edad más frecuente de donación está entre 18 y 30 años (Instituto Nacional de Salud, 2017). A su vez, esta información puede estar relacionada con la distribución de las ocupaciones, en las cuales, se registró mayor proporción de operarios, vendedores de productos y servicios y estudiantes. Con respecto a la frecuencia de donación en operarios, se puede deber a la mayor facilidad de acercamiento a los centros de donación, debido a su jornada laboral, con mayor disponibilidad de tiempo. También, se halló una mayor proporción de donantes residentes en Montería, lo que es concordante con el área de mayor influencia del banco de sangre.

Por otra parte, se halló que la reactividad global (acumulada) con las pruebas de tamización fue de $6,25 \%$, debido a la proporción de resultados positivos; esta cifra fue mucho menor en las confirmatorias, con mayor ocurrencia de $T$. pallidum, T. cruzi y Hepatitis B (Tabla 2). Estos resultados coinciden con un estudio en Medellín, en donde se reportó prevalencia con pruebas tamiz de 3,3\% y en las pruebas confirmatorias, bajó a 0,9\% (Patiño Bedoya et al. 2012). Estas diferencias pueden estar relacionadas con la especificidad de las pruebas tamiz, que pueden ocasionar falsos positivos por diversas patologías asociadas a los marcadores de este estudio, como la tiroiditis de Hashimoto y artritis reumatoidea y a la presencia de parásitos distintos a los tamizados o a la contaminación microbiana, lo cual, podría generar reacciones cruzadas. Por lo anterior, aunque se hace necesaria la sensibilidad mayor del $95 \%$ para validar la prevalencia de las pruebas tamiz, es recomendable la implementación de técnicas con mayor especificidad y, de ese modo, evitar el descarte de unidades sanguíneas, el diferimiento de donantes y las subsecuentes pérdidas económicas para el banco de sangre.

Los resultados de la frecuencia de reactividad global con las pruebas tamiz son mayores que los reportes del INS en el 2016, en los cuales, Córdoba reporta 4,04\%, mientras que el promedio nacional que fue de 3,86\% (Instituto Nacional de Salud, 2017). Estos datos varían con la prueba confirmatoria de este estudio, con la que se obtuvo una prevalencia mucho menor, al tiempo que da cuenta de la particularidad del banco de sangre estudiado, donde se halló una mayor proporción de descarte frente al promedio departamental.

Asimismo, estos resultados son diferentes a los de otra investigación realizada en Montería, en la que se encontró una prevalencia global de 2,3\% en población donante (Pérez \& Máttar, 2003). En Ibagué (Colombia), se halló una prevalencia global, en este mismo tipo de población, de 1,55\%, en 1.420 unidades captadas (Daza Bolaño et al. 2016). Estos resultados muestran una tendencia de diferimientos de donantes, que oscila entre 1,0 y $3,0 \%$, lo que representa una cifra considerable, dada las condiciones de exigencia de selección de los donantes.

La infección con mayor frecuencia de detección fue T. pallidum, lo que es consistente con varios autores, quienes han reportado prevalencias similares; por ejemplo, en un estudio en África, se reportó prevalencia para sífilis de 0,49\% (Fessehaye et al. 2011); 0,72\%, en una investigación en Perú (Concepción Zavaleta et al. 2014) y, en Colombia, se han reportado datos de 1,9\% (Cruz B. et al. 2013). Además, para 2016, en Córdoba, la sífilis presentó una frecuencia en sangre total de 1,7\% y en aféresis, de 1,68\% (Instituto Nacional de Salud, 2017).

En este punto es oportuno precisar que este estudio y otros desarrollados en Colombia, generalmente, reportan la prevalencia de la infección, con base en el número de donantes atendidos y no con base en una cifra que se aproxime más a una prevalencia poblacional por 100.000 donaciones. En este orden de ideas, en esta población, la prevalencia (con pruebas confirmatorias) de infección por cada 100.000 do- 
naciones fue de 45, en VHC;136, para VIH; 50, en VHBHbsAg; 319, en VHB anti-HBc; 78, en HTLV I/II; 387, en T. cruzi y 427, en T. pallidum, lo de denota un alto riesgo poblacional.

En la tabla 3, se observan las prevalencias específicas del virus de la hepatitis $\mathrm{B}$ y el virus de la hepatis $\mathrm{C}$, con las razones de prevalencia que comparan la positividad, según algunas características demográficas y hematológicas. La relevancia de las razones de prevalencia es identificar posibles factores asociados, los subgrupos con mayor frecuencia del evento, al tiempo que el intervalo de confianza de esta medida de asociación da cuenta del grado de precisión de tal estimación, así como su significación estadística (dependiendo de la inclusión del 1,0 o valor nulo para las asociaciones exploradas).

Para el Anti-HBc, se identificaron, como factores asociados, el grupo etario, el lugar de residencia y la ocupación, mientras que, para el HBsAg, no se identificó ninguno. La prevalencia de Anti-HBc fue incrementándose según el grupo etario; en los donantes de 51 a 68 años fue 4,6 veces la reportada en los de 18 a 20 años. Para los residentes por fuera del departamento de Córdoba, la prevalencia fue dos veces la hallada en los donantes de Montería. Según la ocupación, la ocurrencia fue menor en estudiantes y en personal de la salud y mayor en trabajadores del sector agropecuario, amas de casa y pensionados. Estos datos, se podrían complementar con un análisis del estado vacunal, para dar cuenta del riesgo poblacional o la efectividad de algunos programas de vigilancia epidemiológica, seguridad sanitaria, educación, prevención e inmunización; por ejemplo, en Cuba, entre 2012-2013, se reportó una cobertura de vacunación contra la Hepatitis B en trabajadores de la salud, del $88,0 \%$, al tiempo que los trabajadores jóvenes tuvieron resultados positivos significativamente más altos para Anti-HBsAg, pese la presentación de un esquema de vacunación completo (Crespo Ramirez et al. 2018).

Entre las prevalencias más bajas en el estudio, se encuentran las de HBsAg y VHC; en contraste, en Asia se han reportado prevalencias en donantes para estos dos marcadores, de $3,2 \%$ y 0,35\%, respectivamente (Varsha et al. 2017). En Colombia, se han publicado prevalencias de $0,21 \%$, para HBsAg y de 0,45\%, para VHC (Gómez et al. 2014).

La infección por VIH solo presentó diferencias significativas según la ocupación, con prevalencias más altas en estudiantes frente a los operarios. En HTLV, se encontraron diferencias significativas por sexo, lugar de residencia y ocupación. Para T. cruzi, la prevalencia en mujeres fue $68 \%$ mayor que en los hombres y más de nueve veces en personas mayores de 40 años, que la hallada en adolescentes de 18 a 20 años (Tabla 4).
En cuanto a T. pallidum, se identificó como uno de los principales factores asociados a la edad, con un aumento proporcional de la infección en la medida que aumenta el grupo etario. También, se halló asociación con el lugar de residencia y la ocupación (Tabla 5).

Al analizar la proporción global (acumulada) de infección, se identificaron, como potenciales, factores asociados el sexo ( $21 \%$ más en mujeres), la edad ( 4,2 veces más alto en los mayores de 50 años), el lugar de residencia (65\% mayor en residentes de municipios ubicados fuera de Córdoba) y la ocupación (mayor en las amas de casa, pensionados, agricultores y ganaderos) (Tabla 5).

Se realizaron modelos de regresión multivariada para VHB, HTLV, T. pallidum e infección global. En hepatitis B (anti$\mathrm{HBc}$ ), se encontró un efecto independiente de la edad con una odds de infección, de 4,29, en los mayores de 50 años, en comparación con los donantes con edades entre 18-20 años; asimismo, se halló una mayor ocurrencia en residentes fuera del departamento de Córdoba. La ocupación también fue uno de los principales factores asociados con el Virus de la Hepatitis B.

Los factores asociados con HTLV fueron el lugar de residencia, con una ocurrencia 1,93 veces mayor en donantes procedentes de municipios fuera de Córdoba, respecto a los que vivían en Montería y la ocupación. Para T. pallidum fue la edad, con una odds de infección de 8,54, en mayores de 50 años, en comparación con los donantes de 18 a 20 años (Tabla 6).

El riesgo de infección global fue más alto en mujeres que en hombres, resultados que llaman la atención, teniendo en cuenta que la mayoría de los estudios revelan que los hombres son el grupo de mayor ocurrencia. Investigaciones reportadas en Cuba muestran un predominio en la positividad de los marcadores en el sexo masculino, al igual que en el grupo de 18-28 años (Ramos Ríos et al. 2014); sin embargo, en una investigación realizada en México, la frecuencia de infección fue mayor en las mujeres con respecto a los hombres para VHB y VHC (Carreto Vélez et al. 2003), posiblemente, relacionado con la conducta sexual, dado que varios donantes hombres confirmaron haber tenido relaciones extramatrimoniales homosexuales y bisexuales. Otros han asociado el aumento en la reactividad de marcadores infecciosos en la población femenina con el incremento en el ejercicio de las libertades sexuales de la mujer contemporánea (Patiño Bedoya et al. 2012). Todo esto pone de manifiesto la necesidad de adelantar investigaciones dirigidas a evaluar las conductas sexuales de los donantes, que permitan rechazar o consolidar diferentes hipótesis. 
Tabla 3. Comparación de las prevalencias de VHB y VHC según variables demográficas hematológicas.

\begin{tabular}{|c|c|c|c|c|c|c|}
\hline \multirow[t]{2}{*}{ Variable } & \multicolumn{2}{|c|}{ VHB- HbsAg } & \multicolumn{2}{|c|}{ VHB (Anti-HBc) } & \multicolumn{2}{|c|}{ VHC (Anti-VHC) } \\
\hline & $\%$ & RP (IC95\%) & $\%$ & RP (IC95\%) & $\%$ & RP (IC95\%) \\
\hline \multicolumn{7}{|l|}{ Sexo } \\
\hline Hombre & 0,14 & 1 & 2,60 & 1 & 0,31 & 1 \\
\hline Mujer & 0,16 & $\begin{array}{c}1,12 \\
(0,51-2,47)\end{array}$ & 2,82 & $\begin{array}{c}1,09 \\
(0,90-1,30)\end{array}$ & 0,47 & $\begin{array}{c}1,48 \\
(0,93-2,36)\end{array}$ \\
\hline \multicolumn{7}{|l|}{ Grupo etario } \\
\hline $18-20$ años $^{\mathrm{a}}$ & 0,12 & 1 & 0,93 & 1 & 0,12 & 1 \\
\hline 21-30 años & 0,11 & $\begin{array}{c}0,90 \\
(0,21-3,91)\end{array}$ & 1,79 & $\begin{array}{c}1,91 \\
(1,13-3,21)^{*}\end{array}$ & 0,39 & $\begin{array}{c}3,09 \\
(0,75-12,6)\end{array}$ \\
\hline $31-40$ años & 0,18 & $\begin{array}{c}1,46 \\
(0,34-6,20) \\
\end{array}$ & 2,64 & $\begin{array}{c}2,82 \\
(1,68-4,72)^{*}\end{array}$ & 0,35 & $\begin{array}{c}2,78 \\
(0,67-11,49) \\
\end{array}$ \\
\hline $41-50$ años & 0,10 & $\begin{array}{c}0,80 \\
(0,16-3,76) \\
\end{array}$ & 3,61 & $\begin{array}{c}3,86 \\
(2,30-6,47)^{* *}\end{array}$ & 0,24 & $\begin{array}{c}1,90 \\
(0,44-8,14) \\
\end{array}$ \\
\hline 51-68 años & 0,21 & $\begin{array}{c}1,69 \\
(0,36-7,93)\end{array}$ & 4,34 & $\begin{array}{c}4,64 \\
(2,74-7,84) * *\end{array}$ & 0,37 & $\begin{array}{c}2,95 \\
(0,67-12,97)\end{array}$ \\
\hline \multicolumn{7}{|l|}{ Residencia } \\
\hline Montería & 0,13 & 1 & 2,33 & 1 & 0,34 & 1 \\
\hline $\begin{array}{l}\text { Otros municipios de } \\
\text { Córdoba }\end{array}$ & 0,15 & $\begin{array}{c}1,18 \\
(0,65-2,14)\end{array}$ & 3,01 & $\begin{array}{c}1,29 \\
(1,13-1,48) * *\end{array}$ & 0,32 & $\begin{array}{c}0,95 \\
(0,64-1,40) \\
\end{array}$ \\
\hline Municipios fuera de Córdoba & 0,27 & $\begin{array}{c}2,15 \\
(0,84-5,49) \\
\end{array}$ & 4,78 & $\begin{array}{c}2,05 \\
(1,65-2,56) * *\end{array}$ & 0,27 & $\begin{array}{c}0,81 \\
(0,33-1,99)\end{array}$ \\
\hline \multicolumn{7}{|l|}{ Ocupación } \\
\hline Operarios & 0,16 & 1 & 2,97 & 1 & 0,27 & 1 \\
\hline $\begin{array}{l}\text { Ventas de productos } \\
\text { y servicios }\end{array}$ & 0,18 & $\begin{array}{c}1,13 \\
(0,58-2,23) \\
\end{array}$ & 2,95 & $\begin{array}{c}0,99 \\
(0,84-1,17) \\
\end{array}$ & 0,36 & $\begin{array}{c}1,32 \\
(0,81-2,16) \\
\end{array}$ \\
\hline Estudiantes & 0,12 & $\begin{array}{c}0,76 \\
(0,31-1,83) \\
\end{array}$ & 1,65 & $\begin{array}{c}0,55 \\
(0,44-0,70) * *\end{array}$ & 0,30 & $\begin{array}{c}1,10 \\
(0,62-1,98) \\
\end{array}$ \\
\hline $\begin{array}{l}\text { Administración y otras } \\
\text { profesiones }\end{array}$ & 0,02 & $\begin{array}{c}0,15 \\
(0,02-1,12)\end{array}$ & 1,74 & $\begin{array}{c}0,59 \\
(0,46-0,75) * *\end{array}$ & 0,37 & $\begin{array}{c}1,33 \\
(0,75-2,39)\end{array}$ \\
\hline Militares & 0,08 & $\begin{array}{c}0,49 \\
(0,12-2,06)\end{array}$ & 1,81 & $\begin{array}{c}0,61 \\
(0,45-0,82) *\end{array}$ & 0,63 & $\begin{array}{c}2,29 \\
(1,30-4,03)^{* *}\end{array}$ \\
\hline Ama de casa y pensionados & 0,06 & $\begin{array}{c}0,40 \\
(0,05-2,96) \\
\end{array}$ & 4,07 & $\begin{array}{c}1,36 \\
(1,06-1,77)^{*}\end{array}$ & 0,45 & $\begin{array}{c}1,64 \\
(0,74-3,63) \\
\end{array}$ \\
\hline Trabajadores de la Salud & 0,10 & $\begin{array}{c}0,62 \\
(0,08-4,59) \\
\end{array}$ & 1,40 & $\begin{array}{c}0,47 \\
(0,28-0,80)^{*}\end{array}$ & 0,20 & $\begin{array}{c}0,73 \\
(0,18-3,00) \\
\end{array}$ \\
\hline Desempleado & 0,32 & $\begin{array}{c}1,97 \\
(0,60-6,47)\end{array}$ & 2,11 & $\begin{array}{c}0,71 \\
(0,45-1,10) \\
\end{array}$ & 0,11 & $\begin{array}{c}0,38 \\
(0,05-2,77) \\
\end{array}$ \\
\hline Agricultura y ganadería & 0,28 & $\begin{array}{c}1,74 \\
(0,41-7,29) \\
\end{array}$ & 4,88 & $\begin{array}{c}1,64 \\
(1,18-2,29)^{* *}\end{array}$ & 0,56 & $\begin{array}{c}2,03 \\
(0,73-5,61) \\
\end{array}$ \\
\hline \multicolumn{7}{|l|}{ Tipo de donación } \\
\hline Donación altruista & 0,14 & 1 & 2,65 & 1 & 0,33 & 1 \\
\hline Aféresis & 0,17 & $\begin{array}{c}1,23 \\
(0,38-3,96) \\
\end{array}$ & 1,87 & $\begin{array}{c}0,70 \\
(0,50-0,99)\end{array}$ & 0,28 & $\begin{array}{c}0,85 \\
(0,35-2,08) \\
\end{array}$ \\
\hline Donante de reposición & 0,47 & $\begin{array}{c}3,41 \\
(0,47-24,6) \\
\end{array}$ & 3,29 & $\begin{array}{c}1,24 \\
(0,60-2,57) \\
\end{array}$ & 0,47 & $\begin{array}{c}1,41 \\
(0,19-10,04)\end{array}$ \\
\hline Otro & 0,0 & ----- & 0,00 & ---- & 0,00 & ---- \\
\hline
\end{tabular}

a Se agrupó bajo rango de edad, para separar los adultos de los adolescentes; este último grupo, según la OMS, incluye personas entre los 10 y 20 años. RP (IC95\%): Razón de prevalencia con su intervalo de confianza del 95\%. Valor p <0,05* $* * \mathrm{Vp}<0,01$. 
Tabla 4. Comparación de las prevalencias de VIH 1 y 2, HTLV I/II y Trypanosoma cruzi, según variables demográficas y hematológicas.

\begin{tabular}{|c|c|c|c|c|c|c|}
\hline \multirow[t]{2}{*}{ Variable } & \multicolumn{2}{|c|}{ VIH 1 y 2} & \multicolumn{2}{|c|}{ HTLV I/II } & \multicolumn{2}{|c|}{$\begin{array}{l}\text { Trypanosoma cruzi } \\
\text { (anti- T. cruzi) }\end{array}$} \\
\hline & $\%$ & RP (IC95\%) & $\%$ & RP (IC95\%) & $\%$ & RP (IC95\%) \\
\hline \multicolumn{7}{|l|}{ Sexo } \\
\hline Hombre & 0,31 & 1 & 0,49 & 1 & 0,40 & 1 \\
\hline Mujer & 0,42 & $\begin{array}{c}1,38 \\
(0,85-2,24) \\
\end{array}$ & 0,93 & $\begin{array}{c}1,89 \\
(1,35-2,65) * * \\
\end{array}$ & 0,67 & $\begin{array}{c}1,68 \\
(1,13-2,49) *\end{array}$ \\
\hline \multicolumn{7}{|l|}{ Grupo etario } \\
\hline 18-20 años & 0,19 & 1 & 0,50 & 1 & 0,06 & 1 \\
\hline 21-30 años & 0,44 & $\begin{array}{c}2,36 \\
(0,74-7,50) \\
\end{array}$ & 0,53 & $\begin{array}{c}1,05 \\
(0,51-2,18)\end{array}$ & 0,41 & $\begin{array}{c}6,51 \\
(0,90-47,0) \\
\end{array}$ \\
\hline 31-40 años & 0,33 & $\begin{array}{c}1,77 \\
(0,54-5,70)\end{array}$ & 0,52 & $\begin{array}{c}1,04 \\
(0,50-2,17)\end{array}$ & 0,32 & $\begin{array}{c}5,17 \\
(0,71-37,6) \\
\end{array}$ \\
\hline 41-50 años & 0,16 & $\begin{array}{c}0,86 \\
(0,24-3,03) \\
\end{array}$ & 0,59 & $\begin{array}{c}1,17 \\
(0,55-2,48)\end{array}$ & 0,62 & $\begin{array}{c}9,99 \\
(1,38-72,3) * *\end{array}$ \\
\hline 51-68 años & 0,21 & $\begin{array}{c}1,12 \\
(0,30-4,23) \\
\end{array}$ & 0,60 & $\begin{array}{c}1,22 \\
(0,54-2,70)\end{array}$ & 0,58 & $\begin{array}{c}9,28 \\
(1,25-68,77) \\
\end{array}$ \\
\hline \multicolumn{7}{|l|}{ Residencia } \\
\hline Montería & 0,31 & 1 & 0,49 & 1 & 0,42 & 1 \\
\hline $\begin{array}{l}\text { Otros municipios de } \\
\text { Córdoba }\end{array}$ & 0,32 & $\begin{array}{c}1,03 \\
(0,70-1,54)\end{array}$ & 0,60 & $\begin{array}{c}1,21 \\
(0,89-1,63) \\
\end{array}$ & 0,42 & $\begin{array}{c}0,99 \\
(0,70-1,40)\end{array}$ \\
\hline Municipios fuera de Córdoba & 0,44 & $\begin{array}{c}1,38 \\
(0,71-2,69)\end{array}$ & 0,99 & $\begin{array}{c}2,00 \\
(1,23-3,28) * *\end{array}$ & 0,60 & $\begin{array}{c}1,44 \\
(0,78-2,67)\end{array}$ \\
\hline \multicolumn{7}{|l|}{ Ocupación } \\
\hline Operarios & 0,28 & 1 & 0,42 & 1 & 0,46 & 1 \\
\hline $\begin{array}{l}\text { Ventas de productos y } \\
\text { servicios }\end{array}$ & 0,26 & $\begin{array}{c}0,92 \\
(0,52-1,59)\end{array}$ & 0,61 & $\begin{array}{c}1,43 \\
(0,97-2,09) \\
\end{array}$ & 0,39 & $\begin{array}{c}0,85 \\
(0,55-1,32) \\
\end{array}$ \\
\hline Estudiantes & 0,51 & $\begin{array}{c}1,81 \\
(1,11-2,92)^{*}\end{array}$ & 0,77 & $\begin{array}{c}1,82 \\
(1,23-2,69) * * \\
\end{array}$ & 0,33 & $\begin{array}{c}0,70 \\
(0,41-1,20)\end{array}$ \\
\hline $\begin{array}{l}\text { Administración y otras } \\
\text { profesiones }\end{array}$ & 0,27 & $\begin{array}{c}0,96 \\
(0,50-1,84) \\
\end{array}$ & 0,42 & $\begin{array}{c}0,98 \\
(0,58-1,66) \\
\end{array}$ & 0,27 & $\begin{array}{c}0,58 \\
(0,31-1,09) \\
\end{array}$ \\
\hline Militares & 0,35 & $\begin{array}{c}1,26 \\
(0,62-2,57)\end{array}$ & 0,39 & $\begin{array}{c}0,93 \\
(0,48-1,80) \\
\end{array}$ & 0,55 & $\begin{array}{c}1,19 \\
(0,67-2,09) \\
\end{array}$ \\
\hline Ama de casa y pensionados & 0,32 & $\begin{array}{c}1,15 \\
(0,45-2,88)\end{array}$ & 1,29 & $\begin{array}{c}3,05 \\
(1,86-4,98) * * \\
\end{array}$ & 0,58 & $\begin{array}{c}1,25 \\
(0,63-2,49)\end{array}$ \\
\hline Trabajadores de la Salud & 0,40 & $\begin{array}{c}1,40 \\
(0,54-3,59)\end{array}$ & 0,80 & $\begin{array}{c}1,89 \\
(0,91-3,91)\end{array}$ & 0,40 & $\begin{array}{c}0,86 \\
(0,32-2,35)\end{array}$ \\
\hline Desempleado & 0,63 & $\begin{array}{c}2,25 \\
(0,96-5,24)\end{array}$ & 0,42 & $\begin{array}{c}0,99 \\
(0,36-2,71)\end{array}$ & 0,32 & $\begin{array}{c}0,68 \\
(0,21-2,15) \\
\end{array}$ \\
\hline Agricultura y ganadería & 0,14 & $\begin{array}{c}0,49 \\
(0,07-3,59)\end{array}$ & 0,70 & $\begin{array}{c}1,64 \\
(0,67-4,06)\end{array}$ & 0,84 & $\begin{array}{c}1,80 \\
(0,79-4,12)\end{array}$ \\
\hline \multicolumn{7}{|l|}{ Tipo de donación } \\
\hline Donación altruista & 0,32 & 1 & 0,55 & 1 & 0,43 & 1 \\
\hline Aféresis & 0,23 & $\begin{array}{c}0,70 \\
(0,26-1,91) \\
\end{array}$ & 0,40 & $\begin{array}{c}0,72 \\
(0,34-1,53) \\
\end{array}$ & 0,28 & $\begin{array}{c}0,65 \\
(0,27-1,59) \\
\end{array}$ \\
\hline Donante de reposición & 0,94 & $\begin{array}{c}2,93 \\
(0,73-11,80)\end{array}$ & 0,94 & $\begin{array}{c}1,71 \\
(0,43-6,86)\end{array}$ & 0,47 & $\begin{array}{c}1,08 \\
(0,15-7,70)\end{array}$ \\
\hline Otro & 0,0 & ---- & 0,0 & ---- & 0,0 & ---- \\
\hline
\end{tabular}

RP (IC95\%): Razón de prevalencia con su intervalo de confianza del 95\%. Valor $\mathrm{p}<0,05 * * * \mathrm{Vp}<0,01$. 
Tabla 5. Comparación de las prevalencias de Treponema pallidum e infección global, según variables demográficas y hematológicas.

\begin{tabular}{|c|c|c|c|c|}
\hline \multirow[t]{2}{*}{ Variable } & \multicolumn{2}{|c|}{$\begin{array}{c}\text { T. pallidum } \\
\text { (anti T. pallidum) }\end{array}$} & \multicolumn{2}{|c|}{ Prevalencia global } \\
\hline & $\%$ & RP (IC95\%) & $\%$ & RP (IC95\%) \\
\hline \multicolumn{5}{|l|}{ Sexo } \\
\hline Hombre & 2,23 & 1 & 6,10 & 1 \\
\hline Mujer & 2,33 & $\begin{array}{c}1,04 \\
(0,85-1,27)\end{array}$ & 7,42 & $\begin{array}{c}1,21 \\
(1,09-1,36) * *\end{array}$ \\
\hline \multicolumn{5}{|l|}{ Grupo etario } \\
\hline $18-20$ años & 0,93 & 1 & 2,80 & 1 \\
\hline 21-30 años & 0,95 & $\begin{array}{c}1,02 \\
(0,60-1,73)\end{array}$ & 4,33 & $\begin{array}{c}1,55 \\
(1,15-2,08) * *\end{array}$ \\
\hline $31-40$ años & 1,78 & $\begin{array}{c}1,91 \\
(1,13-3,21) *\end{array}$ & 5,87 & $\begin{array}{c}2,09 \\
(1,55-2,81) * *\end{array}$ \\
\hline $41-50$ años & 3,47 & $\begin{array}{c}3,71 \\
(2,22-6,23) * *\end{array}$ & 8,25 & $\begin{array}{c}2,94 \\
(2,19-3,96) * *\end{array}$ \\
\hline $51-68$ años & 6,52 & $\begin{array}{c}6,97 \\
(4,15-11,7) * *\end{array}$ & 11,85 & $\begin{array}{c}4,23 \\
(3,12-5,70) * *\end{array}$ \\
\hline \multicolumn{5}{|l|}{ Residencia } \\
\hline Montería & 1,95 & 1 & 5,67 & 1 \\
\hline $\begin{array}{l}\text { Otros municipios de Cór- } \\
\text { doba }\end{array}$ & 2,84 & $\begin{array}{c}1,45 \\
(1,26-1,67) * *\end{array}$ & 7,21 & $\begin{array}{c}1,27 \\
(1,17-1,38) * *\end{array}$ \\
\hline Municipios fuera de Córdoba & 3,13 & $\begin{array}{c}1,80 \\
(1,37-2,35) * *\end{array}$ & 9,40 & $\begin{array}{c}1,65 \\
(1,42-1,92) * *\end{array}$ \\
\hline \multicolumn{5}{|l|}{ Ocupación } \\
\hline Operarios & 2,54 & 1 & 6,69 & 1 \\
\hline $\begin{array}{l}\text { Ventas de productos y ser- } \\
\text { vicios }\end{array}$ & 2,83 & $\begin{array}{c}1,11 \\
(0,94-1,32) \\
\end{array}$ & 7,06 & $\begin{array}{c}1,05 \\
(0,95-1,17)\end{array}$ \\
\hline Estudiantes & 0,98 & $\begin{array}{c}0,38 \\
(0,28-0,51) * * \\
\end{array}$ & 4,33 & $\begin{array}{c}0,64 \\
(0,56-0,74) * *\end{array}$ \\
\hline $\begin{array}{l}\text { Administración y otras pro- } \\
\text { fesiones }\end{array}$ & 1,42 & $\begin{array}{c}0,56 \\
(0,43-0,73) * *\end{array}$ & 4,41 & $\begin{array}{c}0,66 \\
(0,57-0,77) * *\end{array}$ \\
\hline Militares & 1,73 & $\begin{array}{c}0,68 \\
(0,50-0,93) *\end{array}$ & 5,36 & $\begin{array}{c}0,80 \\
(0,67-0,95) *\end{array}$ \\
\hline Ama de casa y pensionados & 3,87 & $\underset{* *}{1,52(1,17-1,98)}$ & 10,14 & $\begin{array}{c}1,52 \\
(1,29-1,78) * *\end{array}$ \\
\hline Trabajadores de la Salud & 1,50 & $\begin{array}{c}0,59 \\
(0,35-0,98)\end{array}$ & 4,51 & $\begin{array}{c}0,67 \\
(0,50-0,90) * *\end{array}$ \\
\hline Desempleado & 2,0 & $\begin{array}{c}0,79 \\
(0,50-1,24)\end{array}$ & 5,68 & $\begin{array}{c}0,85 \\
(0,65-1,10) \\
\end{array}$ \\
\hline Agricultura y ganadería & 2,65 & $\begin{array}{c}1,04 \\
(0,66-1,64)\end{array}$ & 9,62 & $\begin{array}{c}1,44 \\
(1,14-1,81) * *\end{array}$ \\
\hline \multicolumn{5}{|l|}{ Tipo de donación } \\
\hline Donación altruista & 2,22 & 1 & 6,27 & 1 \\
\hline Aféresis & 2,72 & $\begin{array}{c}1,22 \\
(0,91-1,62)\end{array}$ & 5,61 & $\begin{array}{c}0,89 \\
(0,74-1,09) \\
\end{array}$ \\
\hline Donante de reposición & 2,35 & $\begin{array}{c}1,05 \\
(0,44-2,52)\end{array}$ & 8,45 & $\begin{array}{c}1,34 \\
(0,86-2,10) \\
\end{array}$ \\
\hline Otro & 0,0 & ---- & 0,0 & ---- \\
\hline
\end{tabular}

RP (IC95\%): Razón de prevalencia con su intervalo de confianza del 95\%. Valor $\mathrm{p}<0,05 * * * V p<0,01$. 
Tabla 6. Modelo de regresión logística binaria (razones de odds), para la prevalencia de VHB, HTLV, T. pallidum e infección global.

\begin{tabular}{|c|c|c|c|c|}
\hline & $\begin{array}{c}\text { VHB } \\
\text { (Anti-HBc) }\end{array}$ & HTLV & $\begin{array}{l}\text { Treponema } \\
\text { pallidum }\end{array}$ & Global \\
\hline Sexo Mujer/Hombre & $\begin{array}{c}1,09 \\
(0,87--1,36) \\
\end{array}$ & $\begin{array}{c}1,38 \\
(0,92-2,09)\end{array}$ & $\begin{array}{c}1,10 \\
(0,85-1,41)\end{array}$ & $\begin{array}{c}1,26 \\
(1,09-1,45)^{* *}\end{array}$ \\
\hline \multicolumn{5}{|l|}{ Grupo etario } \\
\hline $\begin{array}{l}21 \text { a } 30 \text { años } / 18 \text { a } 20 \\
\text { años }\end{array}$ & $\begin{array}{c}1,91 \\
(1,12-3,26)^{*}\end{array}$ & $\begin{array}{c}1,25 \\
(0,59-2,66)\end{array}$ & $\begin{array}{c}1,15 \\
(0,66-1,99)\end{array}$ & $\begin{array}{c}1,73 \\
(1,26-2,37) * *\end{array}$ \\
\hline $\begin{array}{l}31 \text { a } 40 \text { años } / 18 \text { a } 20 \\
\text { años }\end{array}$ & $\begin{array}{c}2,76 \\
(1,59-4,78) * *\end{array}$ & $\begin{array}{c}1,45 \\
(0,65-3,27)\end{array}$ & $\begin{array}{c}2,27 \\
(1,28-3,99) * *\end{array}$ & $\begin{array}{c}2,46 \\
(1,77-3,41) * *\end{array}$ \\
\hline $\begin{array}{l}41 \text { a } 50 \text { años } / 18 \text { a } 20 \\
\text { años }\end{array}$ & $\begin{array}{c}3,64 \\
(2,09-6,34) * *\end{array}$ & $\begin{array}{c}1,62 \\
(0,70-3,73)\end{array}$ & $\begin{array}{c}4,4 \\
(2,51-7,86) * *\end{array}$ & $\begin{array}{c}3,48 \\
(2,50-4,84) * *\end{array}$ \\
\hline $\begin{array}{l}51 \text { a } 68 \text { años } / 18 \text { a } 20 \\
\text { años }\end{array}$ & $\begin{array}{c}4,29 \\
(2,44-7,55) * *\end{array}$ & $\begin{array}{c}1,60 \\
(0,66-3,88)\end{array}$ & $\begin{array}{c}8,54 \\
(4,81-15,15) * *\end{array}$ & $\begin{array}{c}5,11 \\
(3,65-7,15) * *\end{array}$ \\
\hline \multicolumn{5}{|l|}{ Lugar de residencia } \\
\hline $\begin{array}{l}\text { Otros municipios de } \\
\text { Córdoba/Montería }\end{array}$ & $\begin{array}{c}1,19 \\
(1,04-1,37) *\end{array}$ & $\begin{array}{c}1,22 \\
(0,90-1,65)\end{array}$ & $\begin{array}{c}1,28 \\
(1,11-1,48) * *\end{array}$ & $\begin{array}{c}1,19 \\
(1,08-1,30) * *\end{array}$ \\
\hline $\begin{array}{l}\text { Municipios por fuera del } \\
\text { departamento /Montería }\end{array}$ & $\begin{array}{c}2,03 \\
(1,61-2,56) * *\end{array}$ & $\begin{array}{c}1,93 \\
(1,17-3,19)^{*}\end{array}$ & $\begin{array}{c}1,49 \\
(1,12-1,97) * *\end{array}$ & $\begin{array}{c}1,60 \\
(1,35-1,90) * *\end{array}$ \\
\hline \multicolumn{5}{|l|}{ Ocupación } \\
\hline Militares/Operarios & $\begin{array}{c}0,85 \\
(0,62-1,17)\end{array}$ & $\begin{array}{c}1,04 \\
(0,52-2,09)\end{array}$ & $\begin{array}{c}1,47 \\
(1,05-2,06) *\end{array}$ & $\begin{array}{c}1,22 \\
(0,99-1,48)\end{array}$ \\
\hline $\begin{array}{l}\text { Trabajadores de la } \\
\text { salud/Operarios }\end{array}$ & $\begin{array}{c}0,49 \\
(0,29-0,85) *\end{array}$ & $\begin{array}{c}1,80 \\
(0,86-3,80) \\
\end{array}$ & $\begin{array}{c}0,71 \\
(0,42-1,20) \\
\end{array}$ & $\begin{array}{c}0,69 \\
(0,51-0,94) *\end{array}$ \\
\hline $\begin{array}{l}\text { Administradores y otras } \\
\text { profesiones /Operarios }\end{array}$ & $\begin{array}{c}0,61 \\
(0,47-0,78) * *\end{array}$ & $\begin{array}{c}0,98 \\
(0,59-1,67)\end{array}$ & $\begin{array}{c}0,63 \\
(0,48-0,83) * *\end{array}$ & $\begin{array}{c}0,68 \\
(0,58-0,80) * *\end{array}$ \\
\hline $\begin{array}{l}\text { Agricultura y ganadería / } \\
\text { Operarios }\end{array}$ & $\begin{array}{c}1,34 \\
(0,94-1,91)\end{array}$ & $\begin{array}{c}1,47 \\
(0,59-3,68)\end{array}$ & $\begin{array}{c}0,68 \\
(0,42-1,09)\end{array}$ & $\begin{array}{c}1,15 \\
(0,89-1,49)\end{array}$ \\
\hline $\begin{array}{l}\text { Ama de casa y } \\
\text { pensionados/ Operarios }\end{array}$ & $\begin{array}{c}1,15 \\
(0,84-1,58)\end{array}$ & $\begin{array}{c}2,27 \\
(1,26-4,08) * *\end{array}$ & $\begin{array}{c}1,21 \\
(0,87-1,68)\end{array}$ & $\begin{array}{c}1,18 \\
(0,96-1,45)\end{array}$ \\
\hline Estudiantes /Operarios & $\begin{array}{c}0,88 \\
(0,67-1,16)\end{array}$ & $\begin{array}{c}2,11 \\
(1,32-3,37) * *\end{array}$ & $\begin{array}{c}1,02 \\
(0,72-1,45)\end{array}$ & $\begin{array}{c}1,05 \\
(0,88-1,25) \\
\end{array}$ \\
\hline Ventas/Operarios & $\begin{array}{c}1,01 \\
(0,85-1,19)\end{array}$ & $\begin{array}{c}1,44 \\
(0,98-2,12)\end{array}$ & $\begin{array}{c}1,17 \\
(0,98-1,39)\end{array}$ & $\begin{array}{c}1,08 \\
(0,97-1,21)\end{array}$ \\
\hline $\begin{array}{l}\text { Desempleados/ } \\
\text { Operarios }\end{array}$ & $\begin{array}{c}0,90 \\
(0,57-1,43)\end{array}$ & $\begin{array}{c}1,09 \\
(0,39-3,00)\end{array}$ & $\begin{array}{c}1,31 \\
(0,82-2,11)\end{array}$ & $\begin{array}{c}1,12 \\
(0,84-1,48)\end{array}$ \\
\hline
\end{tabular}

Valor $\mathrm{p}<0,05^{*}$, Valor $\mathrm{p}<; 0,01^{* *}$

De acuerdo con los resultados obtenidos en este trabajo, la prevalencia de Infecciones Transmisibles por vía Transfusional es baja frente a estudios previos, pero significativa, ya que es evidente que existe un riesgo para los pacientes y aunque en la actualidad gran parte de la nueva tecnología ha enfocado sus avances en disminuir el tiempo de detección y se han establecidos tiempos de diferimientos largos con relación al periodo de ventana inmunológica, la práctica rutinaria de medidas coercitivas que condicionan una cirugía, hospitalización o visita a un familiar o amigo enfermo para lograr que las personas donen sangre, compromete la confiabilidad en las respuestas a las encuestas de salud, usadas para excluir a individuos de alto riesgo y la seguridad de la sangre obtenida en estas condiciones.

Entre las limitaciones del estudio, se pueden resaltar el sesgo temporal de todos los estudios transversales, lo cual, implica que las asociaciones estadísticas tienen un carácter explora- 
torio y no causal. En adición, se debe precisar que los intervalos de confianza presentaron una alta amplitud, lo que puede dificultar algunas comparaciones entre subgrupos de donantes.

A pesar de estas limitaciones, presenta múltiples ventajas, como el trabajar con datos poblacionales, exponer la magnitud de la ocurrencia de infecciones que no se incluyen rutinariamente en los programas de vigilancia epidemiológica activa de Colombia, identificar grupos con mayor ocurrencia de infecciones, además de la generación de hipótesis, que permiten abrir campo a más investigaciones, relacionadas con estos factores.

Lo expuesto permite concluir que la prevalencia de infecciones fue baja en comparación con investigaciones previas discutidas en los párrafos anteriores; sin embargo, el hallazgo de subgrupos con prevalencias más altas, como las mujeres, personas de mayor edad, residentes fuera de Córdoba, amas de casa, pensionados, administradores, militares y trabajadores de la salud, permite orientar nuevas investigaciones e intervenciones de vigilancia epidemiológica, así como focalizar las acciones educativas desde el banco de sangre.

Conflicto de intereses: El manuscrito fue preparado y revisado con la participación de todos los autores, quienes declaramos que no existe ningún conflicto de intereses que ponga en riesgo la validez de los resultados presentados.

\section{REFERENCIAS}

1. BLEJER, J.L.; CARRERAS VESCIO, L.A.; SALAMONE, H.J. 2002. Riesgo de transmisión de infecciones por vía transfusional. Rev Medicina. (Buenos Aires). 62(3):259-278.

2. CARRETO-VÉLEZ, M.A.; CARRADA-BRAVO, T.; MARTÍNEZ-MAGDALENO, A. 2003. Seroprevalencia de VHB, VHC y VIH en donadores de sangre en Irapuato, México. Rev. Salud Publica. (Mexico). 45(5):S690S3.

3. CONCEPCIÓN ZAVALETA, M.; CONCEPCIÓN URTEAGA, L.; MARCHENA AVILA, M.; ESTRADA ALVA, L. 2014. Frecuencia de marcadores serológicos de infecciones transmisibles por transfusión sanguínea en donantes voluntarios en un hospital de Trujillo, Perú. Rev. Cuerpo méd. HNAAA. (Perú). 7(3):18-22.

4. CRESPO RAMIREZ, E.; GUANCHE GARCELL, H.; MARQUEZ FERRER, A. 2018. Estado inmunológico contra hepatitis B en trabajadores de la salud en hospital comunitario de Qatar. Rev. Ciencias Médicas Pinar del Rio. (Cuba). 22(2):365-372.

5. CRUZ B., H.F.; PATIÑO P., A.; MADERO R., J. 2013. Tamizaje para sífilis en donantes de sangre y reactividad simultánea con otros marcadores en la Fundación Hematológica Colombia. Rev. Col. Enfermería. 8(8):46-52.

6. CRUZ B., H.F.; MORENO C., J.E. 2016. Seroprevalencia de tamizaje de Chagas y factores asociados a coinfección en un banco de sangre de Colombia durante 2006-2011. Rev. Méd. Risaralda. 21(1):26-30.

7. DAZA BOLAÑO, N.; SANCHEZ JAIMES, M.; VANEGAS ESTÉVEZ, T.; ORTEGA HERNANDEZ, I. 2016. Prevalencia de infecciones en donantes de sangre en la Universidad Industrial de Santander versus parques de la ciudad de Bucaramanga, 2014. Rev MED. UIS. (Colombia). 29(3):55-60.

8. FESSEHAYE, N.; NAIK, D.; FESSEHAYE, T. 2011. Transfusion transmitted infections - a retrospective analysis from the National Blood Transfusion Service in Eritrea. Rev. Pan Afr. Med. J. 9(40):1-6.

9. GIRALDO-VALENCIA, E.C.; MORALES-GALLO, M.E.; MAYA-GUERRERO, M.A.; RENDÓN-CASTRILLÓN, L.E.; CARDONA ARIAS, J.A. 2015. Prevalencia de marcadores de infecciones transmisibles y su relación con variables demográficas en un banco de sangre de Antioquia Colombia, 2010-2013. Rev. CES Med. (Colombia). 29(1):59-74.

10. GÓMEZ, L.A.; PEÑUELA, O.; HIGUERA, F. 2014. Prevalence of antibodies against transfusion-transmissible infections (TTI) in blood donors from the Colombian eastern region. Rev.Clin.Lab. 60(5):869-871.

11. INSTITUTO NACIONAL DE SALUD, INS. 2013. Guía para la selección de donantes de sangre en Colombia 2012. 72p.

12. INSTITUTO NACIONAL DE SALUD. 2017. Informe anual de la red nacional de bancos de sangre 2016. 35p.

13. MANDAL, R.; MONDAL, K. 2016. Transfusion transmissible infections among blood donors froma sub-Himalayan rural tertiary care centre in Darjeeling, India. J. Trad. Complemen. Med. 6(1):224-229.

14. MINISTERIO DE SALUD PÚBLICA. REPÚBLICA DE COLOMBIA. 1996. Resolución número 00901 de 1996. Bogotá. 
15. MINISTERIO DE SALUD Y PROTECCIÓN SOCIAL. REPÚBLICA DE COLOMBIA. 2014. Resolución número 000437 de 2014. Bogotá.

16. ORGANIZACIÓN INTERNACIONAL DEL TRABAJO, OIT. 1958. Clasificación internacional uniforme de ocupaciones. [Actualizada desde 1996-2018]. Disponible desde Internet en: http://www.ilo.org/public/libdoc/ilo/1958/58B09_81_span.pdf (con acceso16/02/2018).

17. ORGANIZACIÓN MUNDIAL DE LA SALUD -OMS-. 2005. 58 a Asamblea Mundial de la Salud. [Actualizada mayo 2005]. Disponible desde Internet en: http://apps.who.int/gb/ebwha/pdf_files/wha58-rec1/ a58_2005_rec1-sp.pdf (con acceso 25/023/2018).

18. ORGANIZACIÓN MUNDIAL DE LA SALUD -OMS-. 2010. Screening donated blood for transfusión - trasmisible infección. [Actualizada desde 2010]. Disponible desde Internet en: http://www.who.int/bloodsafety/ ScreeningDonatedBloodforTransfusion.pdf (con acceso10/02/2018).

19. ORGANIZACIÓN PANAMERICANA DE LA SALUD. 2017. Suministro de sangre para transfusiones en los países de Latinoamérica y del Caribe, 2014 y 2015. Washington D.C.

20. PATIÑO BEDOYA, J.A.; COTÉS MÁRQUEZ, M.M.; CARDONA ARIAS, J.A. 2012. Seroprevalencia de marcadores de infecciones transmisibles por vía transfusional en banco de sangre de Colombia. Rev. Saúde Pública. (Brasil). 46(6):950-959.

21. PÉREZ F., D.; MÁTTAR V., S. 2003. Prevalencia de marcadores infecciosos en el banco de sangre del hospi- tal San Jerónimo de Montería: 1996 - 2001. Infectio. (Colombia). 7(1):15-20.

22. RAMOS RIOS, M.A.; HERNÁNDEZ DÍAZ, E.; MIRANDA GÓMEZ, O.; PREVOT CAZÓN, V.; BOCOURT RODRIGUEZ, A.; SORÁ PÉREZ, D. 2014. Incidencia de marcadores serológicos en donantes de sangre. Rev. Cub. Med. Mil. (Cuba). 43(4):441-48.

23. SUÁREZ G., E.L.; ERANIDE, L.; DE FREITAS F., H.A.; HANNAOUI R., E.J; GÓMEZ A., L.J. 2007. Prevalencia de enfermedades infecciosas de transmisión sanguínea en donantes que asisten al Banco de Sangre del Hospital Universitario "Antonio Patricio de Alcalá”, Cumaná, Estado Sucre. Rev. Kasmera. (Cubana). 35(1):56-64.

24. VARSHA G., S.; NANDKUMAR M., D.; PRADEEP A., G. 2017. Seroprevalence of HIV, HBV, HCV and Syphilis among Blood Donors in Western Maharashtra and a Newer Proposed Donor Screening Algorithm. J. Blood Disord.Transfus. 8(3):1-3.

25. XIE, D.D.; LI, J.; CHEN, J.T.; EYI, U.M.; MATESA, R.A.; OBONO, M.M.; EHAPO, C.S.; YANG, L.Y.; YANG, H.T.; LIN, M. 2015. Seroprevalence of human Immunodeficiency virus, hepatitis $B$ virus, hepatitis $C$ virus, and Treponema pallidum infections among blood donors on Bioko Island, Equatorial Guinea. PLoS ONE 10(10): e0139947.

Recibido: Marzo 29 de 2018

Aceptado: Septiembre 25 de 2018

\section{Cómo citar:}

Ruiz Mendoza, L.; Villegas Gracia, R.; Cardona Arias, J. 2018. Prevalencia de agentes transmisibles por transfusión y factores asociados en un banco de sangre de Córdoba- Colombia 2014-2016. Rev. U.D.C.A Act. \& Div. Cent. 21(2):297-308. https:// doi.org/10.31910/rudca.v21.n2.2018.969 